\title{
Special aspects of using a new non-stationary random processes analysis approach in the conditions of a priori uncertainty of the useful signal function
}

\author{
Vladimir Marchuk ${ }^{1, *}$ \\ ${ }^{1}$ Don State Technical University, 344000 Rostov-on-Don, Russia
}

\begin{abstract}
The paper considers the use of a new method of signal processing in the time domain under conditions of a limited amount of a priori information about the useful signal function and the statistical characteristics of additive noise. Research have shown its high efficiency in processing signals both local and global, using it to detect anomalous measurements, eliminating the systematic component in the case of a onesided law of the distribution of additive noise and a number of others.
\end{abstract}

\section{Introduction}

Nowadays, the amount of information transmitted by radio systems is constantly growing, which is determined by the increase in the number of measured parameters that are used to monitor and control complex industrial facilities. The complexity of scientific research and, as a consequence, the expansion of the measured parameters increases the measurement information flow, the processing of which is complicated due to the uniqueness of the studies and the limited amount of a priori information about the measured function of the useful signal and statistical characteristics.

Most telemetry implementations relate to non-stationary signals of additive nature. Series of measurement results can be considered as a limited set of random variables of some non-stationary random signal, which can be represented as the sum of some useful component and random, obeying some probabilistic distribution.

In practice, use of the methods considered in the works of David G., Perevertkin S.M., Bendat J., Andersen T., Wiener N., Kalman R.E., Brandt Z., Levina B.R., Tsvetkova E.I., Tikhonova V.I., Krinetskogo E.I., Fomina A.F., Ayvazyana S.A., Likhareva V.A. and a number of others, it is possible only if there is the necessary amount of a priori information about both useful and random components. Otherwise, their effectiveness is substantial reduced, which reduces the reliability of the analysis. Thereby, despite a huge number of works on this topic, computer processing of data is carried out either by visual analysis, or by simple methods, such as the moving average method and its modifications. This is especially evident when processing measurement results, which are represented by the only limited implementation of the measured process with insufficient a priori information.

* Corresponding author: marchuk@sssu.ru 
In the general case, a priori information about the function of the measured process is extremely small, especially when processing the results of unique measurements presented by a single implementation. There are quite a lot of such examples in practice. In this case, the useful signal function, which is a function of time or some other variables, is a priori unknown, but can be represented approximately as a linear combination of known time functions [1].

We consider the function $S(t)$ smooth according to Anderson [1]. By a smooth function we mean a function that is reasonably well approximated by a polynomial no higher than the second degree on some (not too small) time interval. This assumption is confirmed by the first Weierstrass theorem and the Taylor theorem. Polynomials approximating the useful signal function do not have to be the same at different intervals, i.e. actually we are talking about piecewise smoothness. At the same time, the assumption of smoothness on any interval allows us to speak approximately about it's smoothness over the entire range of definition.

In the works of Anderson T., Peregudov V.I. [1,2] was proved that the obtained leastsquares unbiased estimate is the best, in case if random errors are uncorrelated and distributed according to the Gaussian law $N(0, \sigma)$, because each element of the coefficient vector estimation has the dispersion that is the smallest among the dispersions of all unbiased estimates of the corresponding coefficient vector element. Anderson T. [1] shows that if the degree of the approximating polynomial is correctly chosen, the coefficient vector estimation also has the above mentioned properties for correlated samples.

However, the least squares method requires a priori information about the approximating function over the entire processing time interval. Perevertkin S.M. in [3] estimates the accuracy properties of the least squares method by modeling processes with a priori known statistical characteristics. The minimum value of the error is achieved in the middle of the implementation. With an increase in the order of the approximating polynomial, dispersion estimate of error increases, reaching at the ends of the implementation a value exceeding dispersion of the random component. With decreasing the order of approximating polynomial, the magnitude of bias in the estimate of the measured signal increases. Therefore, to minimize the error in the approximation by the least squares method of the measured function, it is necessary to know exactly the order of approximating polynomial and statistical characteristics of the measurement errors.

As the analysis of literature shows, in the presence of a single implementation of a random non-stationary signal with nonparametric a priori uncertainty, the data is processed after a preliminary partition of the initial implementation into quasistationary sections with their subsequent approximation by a low degree polynomial (usually not higher than the second). Stationarity of the measured data can be checked by parametric and nonparametric methods of the theory of statistical solutions $[3,4]$.

\section{Problem definition}

The analysis of non-parametric Kendall statistics in [3] shows that with its help it is possible not only to obtain answers with a certain reliability about the quasistationarity of the implementation segment of a random process of fixed length, but also to partition this interval into intervals of approximation of a variable length, depending on the behavior of the useful component.

However, it should be noted that when using Kendall statistics when processing a useful signal that does not start with the first measurement, the interval value can be so increased due to diverging threshold values that the selection error can be quite large. On the other hand, if the measured function increases sharply and the dispersion of the noise component 
is large, then dividing into sufficiently small intervals with their subsequent approximation also leads to an increase in the separation error.

In [6], it was analytically proved and experimentally confirmed that when the function of the useful signal is a priori unknown, or it cannot be represented as a linear combination of known functions, separation methods are used that are based on preliminary dividing the initial process into intervals, followed by approximation by the least squares of the estimation of the useful signal on the obtained segments by some known function. The estimate of the useful signal function obtained in this way is the sum of the estimates determined at each of the intervals and represents some broken curve. In this case, influence of associated interference is significantly attenuated. The obtained results allow us to conclude that for each measured function there is an optimal number of partitions at which the approximation error reaches its lowest value, as shown in fig. 1.

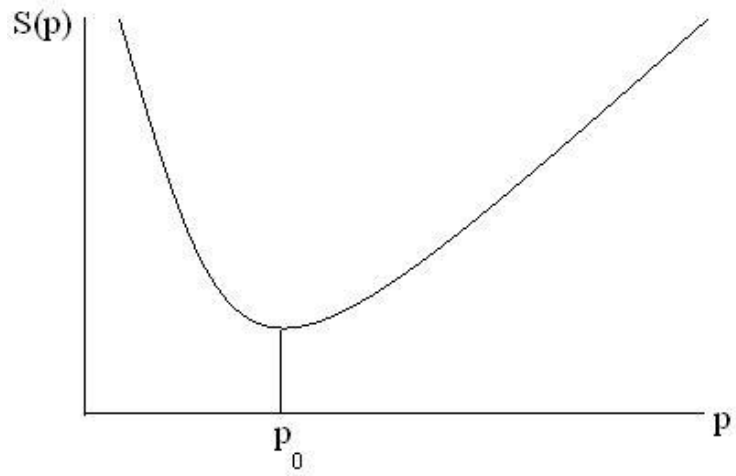

Fig. 1. Determining the sample partitions number of measurement results.

The optimal number of partitions depends on the function of the useful component and the intervals of the partitions may contain a different number of measurement results. Under conditions of a priori nonparametric indetermination, it is impossible to determine the values and number of partition intervals using known methods.

\section{Problem solution}

Using the developed method of multiplying estimates of the useful component (patent No. 2207622) allows us to extract, the useful component under conditions of nonparametric a priori uncertainty, and the resulting estimate can be continuous and asymptotically optimal, which was proved in [6]. The structural diagram of the method of propagation of estimates (MPE) is shown in Fig. 2. In a first approximation, the number of multiplied estimates of the emitted useful signal $(P)$ can be determined from the classical approach of averaging over the volume of realizations: the larger the number of multiplied realizations of the useful signal, the more accurate is the estimate of the useful component [1,5], which is true only when the sample size tend to infinity.

The realization of this condition with a limited sample size allows to get a very specific number of breeding, which is defined and proved analytically in [6] and which is described by the expression:

$$
P(N, R, L)=\left\{\begin{array}{lll}
0 & \text { where } N<L(R+1) \\
N-(R+1) L+1 & \text { where } L(R+1) \leq N<L(R+2)-1 \\
{\left[\frac{N-2 L+1}{R}\right]} & \text { where } N \geq L(R+2)-1
\end{array}\right.
$$


where $P$ - number of breeding; $N$ - sample size; $R$ - number of sample splits ; $L-$ minimum partition interval width.

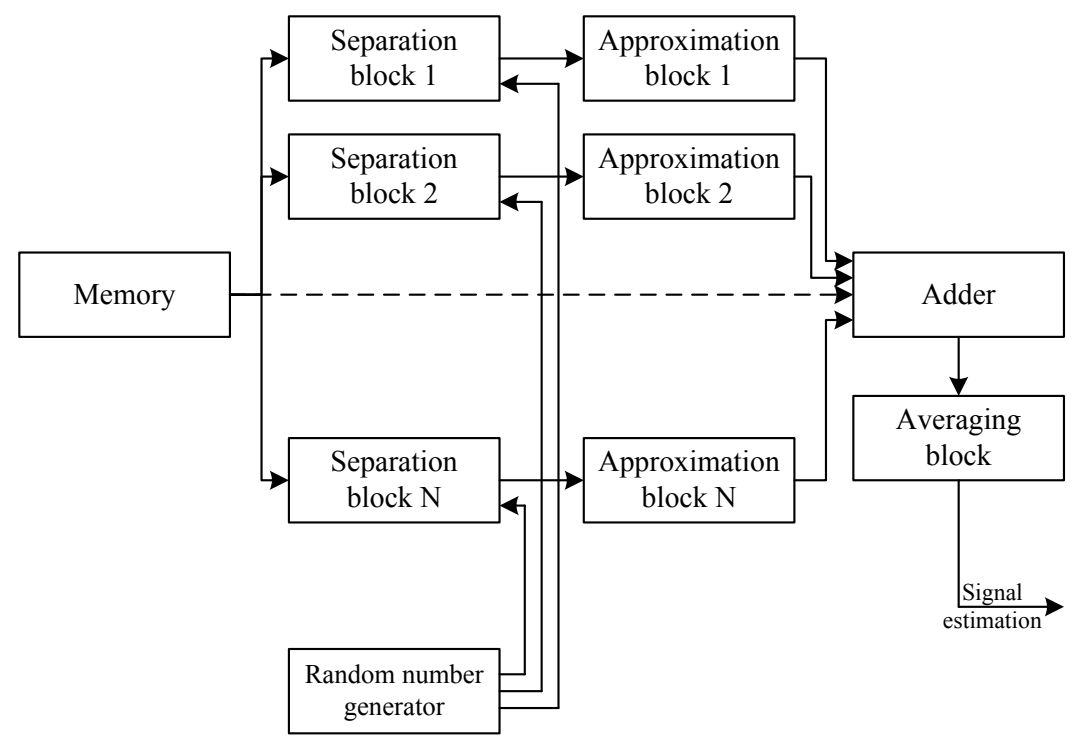

Fig. 2. Flowchart of an algorithm that implements the method of propagating estimates of the useful component.

The resulting expression allows us to determine a functional relationship between the main parameters of the MPE method, and experimental studies based on the experimentalstatistical method - simulation modeling have confirmed the theoretical results obtained. The dependence of $m_{M S D}$ and $\sigma_{m_{M S D}}$ was considered, and their values were calculated from 1000 realizations of length $\mathrm{N}=100$. The results of experimental statistical analysis are presented in Fig. 3.

An analysis of the obtained experimental results shown in Fig. 3 shows that the use of LSM, with the a priori known function of a useful signal (1 partition), the smallest value of $m_{M S D}$ is 0.0124 . With equable partitioning into intervals, the error increases (curve 1) and, with increasing $\mathrm{R}$, asymptotically tends to the standard deviation of the noise.

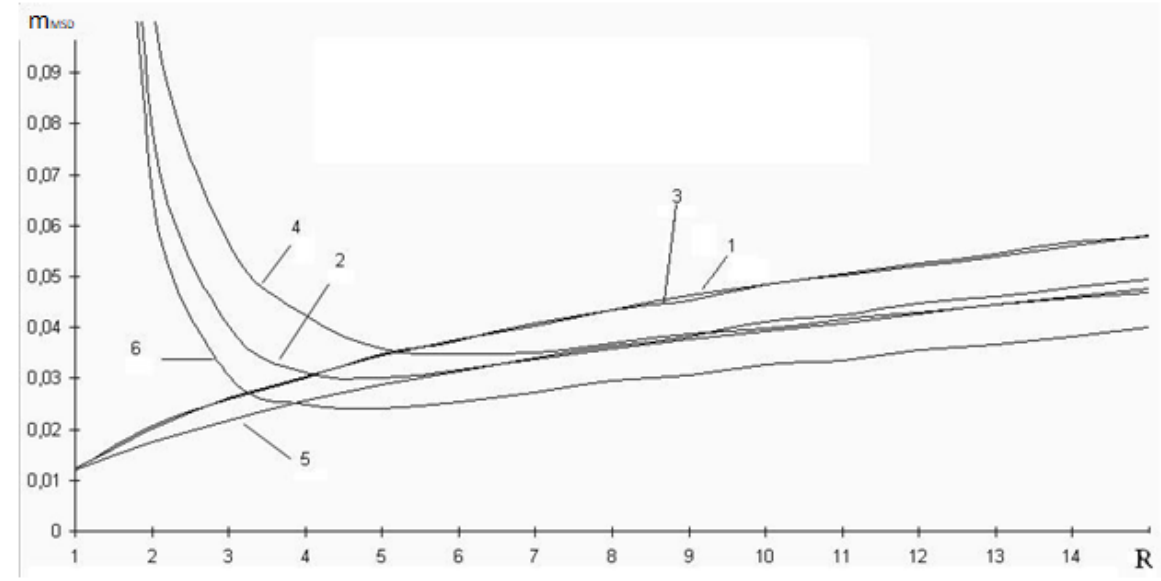

Fig. 3. Dependence $m_{M S D}=\mathrm{f}(\mathrm{R})$ for a priori known and unknown function of useful component. 
In case, if there is no a priori information about the useful component function (the model is a polynomial of the second degree, and the approximation is a polynomial of the first degree), the form of the dependence $=f(R)$, (where $\mathrm{R}$ is the number the original sample separations) of measurement results sharply changes (curve 2). When approximating the entire sample, the error has a maximum value, and with an increase in the partition number $R$, the value $m_{M S D}$ decreases, while the minimum value is reached at $\mathrm{R}=5$ and amounts to 0.03 . Increase in $\mathrm{R}$ leads to an increase in $m_{M S D}$, which also asymptotically tends to the value of standard deviation of noise.

The use of the (MPE) method (the partition is carried out according to a random uniform law) with an approximation on each coverage by a polynomial of the second degree (graph 3) almost repeats the results obtained by uniformly dividing the original sample into approximation intervals (the difference is not more than $0.2 \%$ ), i.e. the partition method (uniform or random) almost does not affect the approximation error. When using the method of multiplying estimates with approximation on each coverage by a polynomial of the first degree (graph 4), the error also has a minimum and with an increase in the number of partitions, curves 3 and 4 almost coincide. Figure 3 (graph 5) shows the results of using the method of multiplying estimates (at $P=15$ ) for approximation on each of the coatings by a polynomial of the second degree (graph 5). In this case, a decrease in the approximation error is observed over the entire range of variation of the parameter $\mathrm{R}$ by an average of $16 \%$. Using the method of multiplying estimates of the useful component allows the processing of measurement results with a limited amount of a priori information about the function of the useful component, while the approximation error asymptotically tends to optimal, which corresponds to the case of using approximation by least-squares method in the presence of a priori information about the function of the useful component.

Consider the dependence $m_{M S D}=f\left(\sigma_{n}\right)$ when using various methods of processing the measurement results, which are presented in Fig. 4. The analysis of the obtained dependences shows that the minimum value of $m_{M S D}$ is achieved using the method of multiplying estimates, and in almost the entire range of variation of the dispersion of the additive noise component. For example, with an increase in the standard deviation of the additive noise component to $\sigma_{n}=0,25$ the method of multiplying estimates has an $m_{M S D}$ value of $8 \%$ less than the moving average method, and an average of 30-70\% less than other methods. Only with $\sigma_{n}=0,3$ the value of $m_{M S D}$ is $8 \%$ higher than when using the moving average method. As in the previous case, if there is a priori information about the dispersion of the additive noise component, it is possible to decrease the value of $m_{M S D}$ by 1.2 times due to a change in the degree of the approximating polynomial on each of the covers.

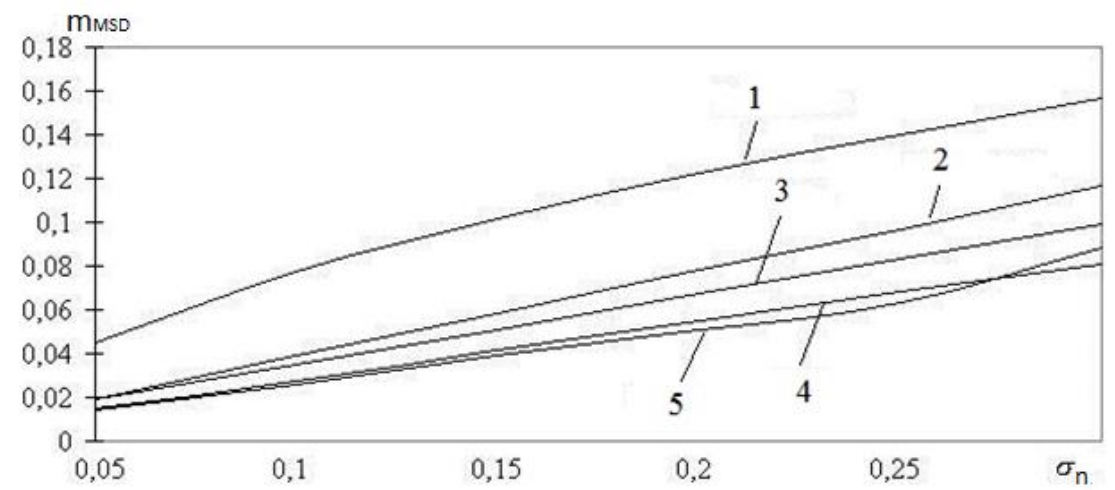

Fig. 4. Dependence $m_{M S D}=f\left(\sigma_{n}\right)$ for various processing methods $(1-$ exponential method, 2 weighted mean method, 3 - median method, 4 - moving average method, 5 - method MPE). 
When conducting research for each of the methods, the optimal parameters were determined and the end effects were not taken into account (methods 2-4). The MPE method was used without preliminary determination of the optimal parameters, i.e. in conditions of a priori insufficiency.

When using the "caterpillar" method, the research results showed a great dependence on a priori information, for example, incorrect determination of the length of the "caterpillar" increases the value of $m_{M S D}$ by an average of 5-15\%, as shown in Fig. 5. Thus, the use of the "caterpillar" method also requires a priori information about the function of the measured process and the statistical characteristics of the additive component for the correct choice of its length.

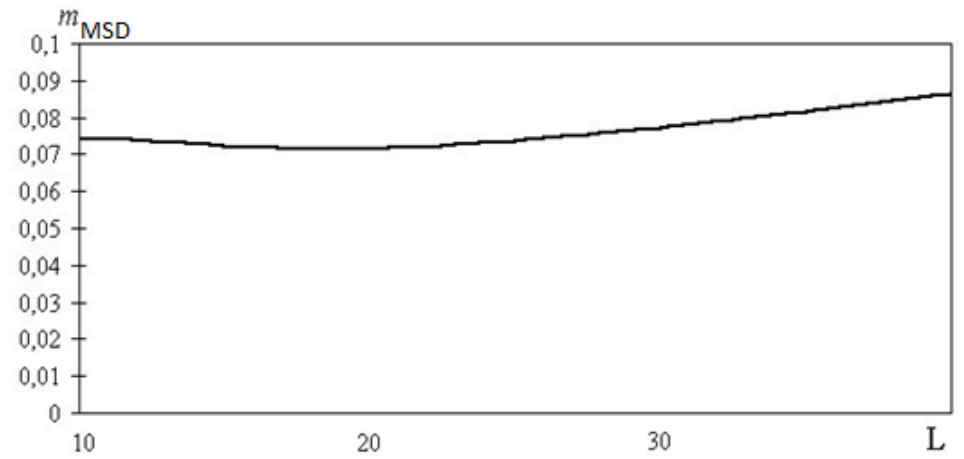

Fig. 5. Dependence $m_{M S D}=f(L)$ for the Gaussian law of the distribution of the additive noise component.

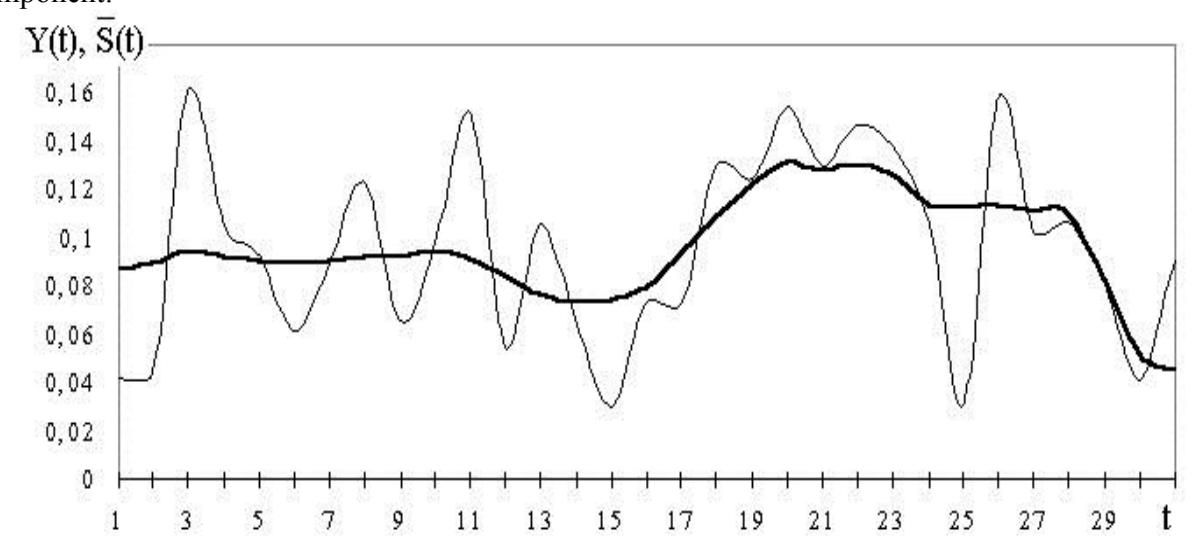

Fig. 6. Fragment of the results of processing by the method of multiplying estimates.

Figure 6 shows a fragment of the evaluation of the useful component function, which passes through the central values of the measurement results. This feature allows you to significantly diversify the scope of the MPE method. One example of this is the processing of measurement results with a one-sided law of the distribution of the random component, shown in Fig. 5, where 1 is the biased estimate of the signal, 2 is the estimate with offset compensation. The resulting systematic component can be compensated by using the proposed methodology based on the MPE method, which is as follows. With the one-sided law of the distribution of additive noise, the probability of the appearance of the smallest value of a random process depends on the volume of the initial implementation of the measurement results, with an increase in which the probability of such a value increasing. The minimum value of remainders determination makes it possible to compensate the useful signal estimation by this value. 


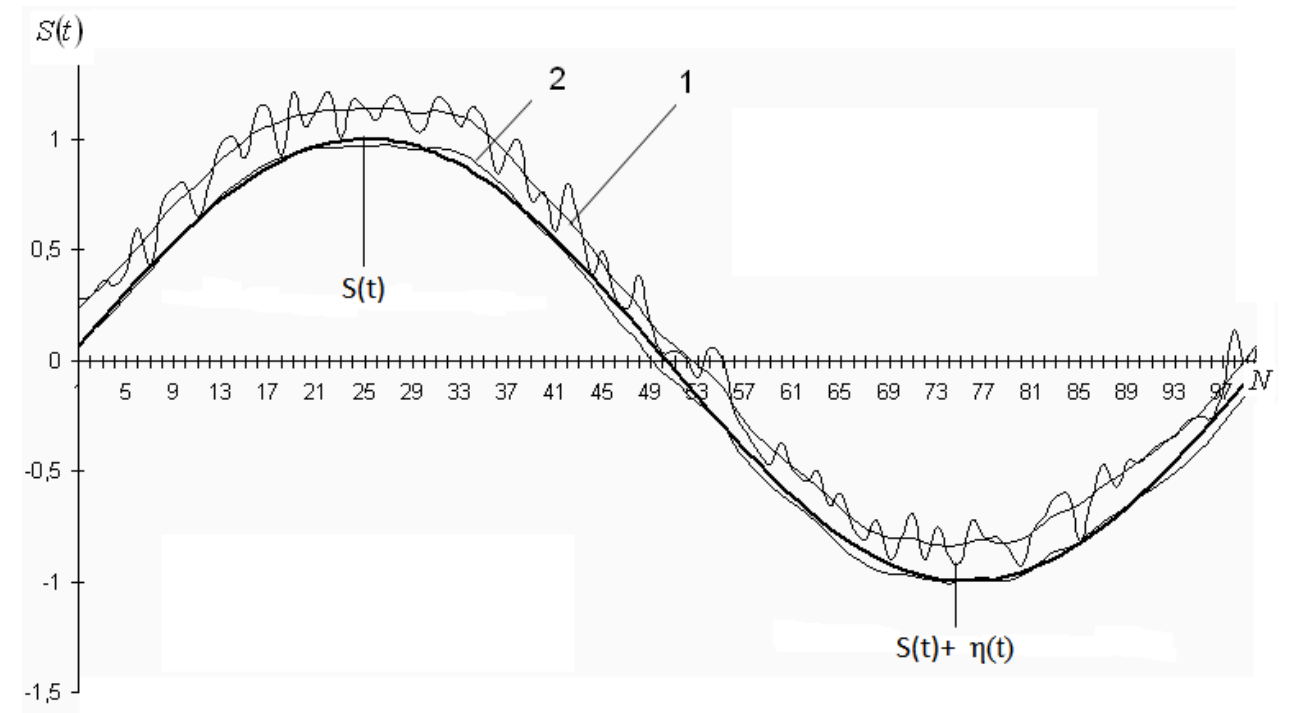

Fig. 7. The processing results of the measurement results with an uncentred additive noise component.

In other words, the higher appearance probability of the minimum values in the remainders, the higher accuracy of the compensation for the bias of the resulting estimate. Research on the processing of measurement results using the proposed bias compensation techniques were carried out for the case of one-sided noise distribution laws, at $\bar{m} \neq 0$.

Figure 8 shows the dependence of the standard error of the selection of the useful component $\sigma_{0}$ on the standard deviation of the additive noise component $\sigma_{n}$ for the case of introducing compensation for the systematic component of the error (graph 1) and without compensation (graph 2) for the case of the same model of the useful signal.

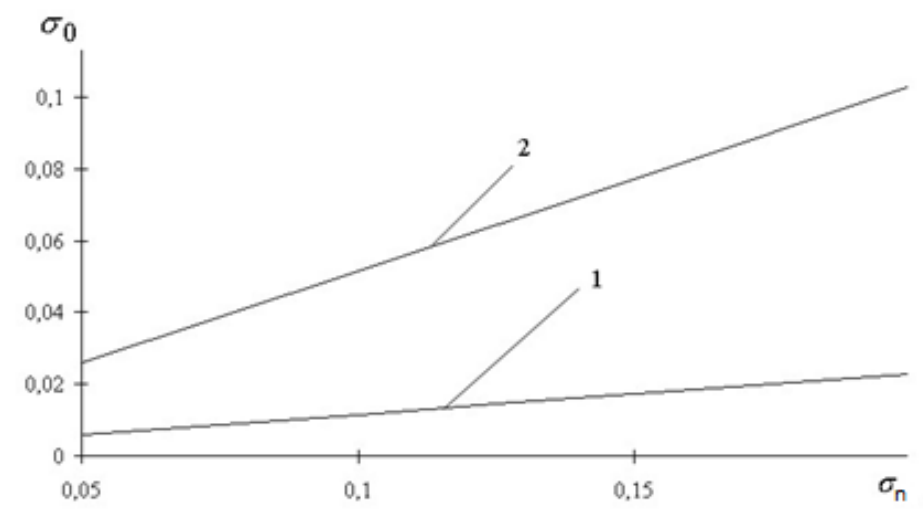

Fig. 8. Graphs of the dependence of $\sigma_{0}=f\left(\sigma_{n}\right)$ with compensation and without compensation of the approximation error (uniform distribution law)

An analysis of the obtained dependences shows that the use of the proposed method allows almost completely compensate for the shift in the estimate of the useful signal function. The mean square error in this case decreases by more than 4 times.

Figure 9 shows $\sigma_{0}=f\left(\sigma_{n}\right)$ dependences for the uniform distribution law of the additive component at $\bar{m} \neq 0$ with compensation of the systematic component (graph 1) and at $\bar{m}=0$ (graph 2), which show high efficiency using the compensation method. The difference in the values of the errors does not exceed $6 \%$. 


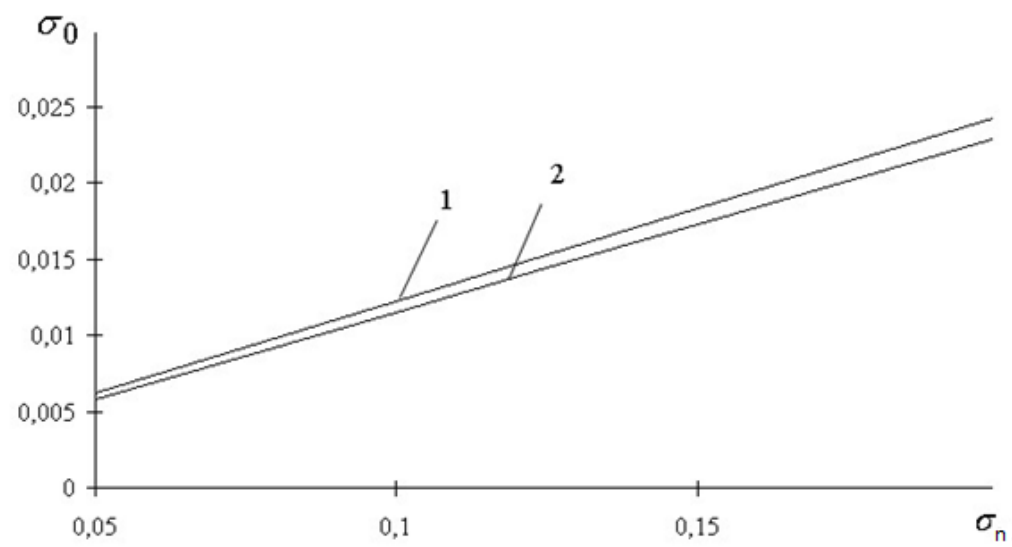

Fig. 9. Dependency graphs $\sigma_{0}=f\left(\sigma_{n}\right)$ at $\bar{m} \neq 0$ and $\bar{m}=0$.

The results of the studies showed that the change in the length of the implementation over a wide range does not affect the performance of the proposed method. The filtering of the additive noise component makes it possible to extract the function of the measured process, which characterizes the investigated transient good enough. The oscillatory process of the transition process can be analyzed to determine its main parameters. In the case, when it is known a priori that the measured value over a larger interval is described by a low polynomial (when a researcher is interested in the trend of the current process), the processing results are presented in Fig. 10.

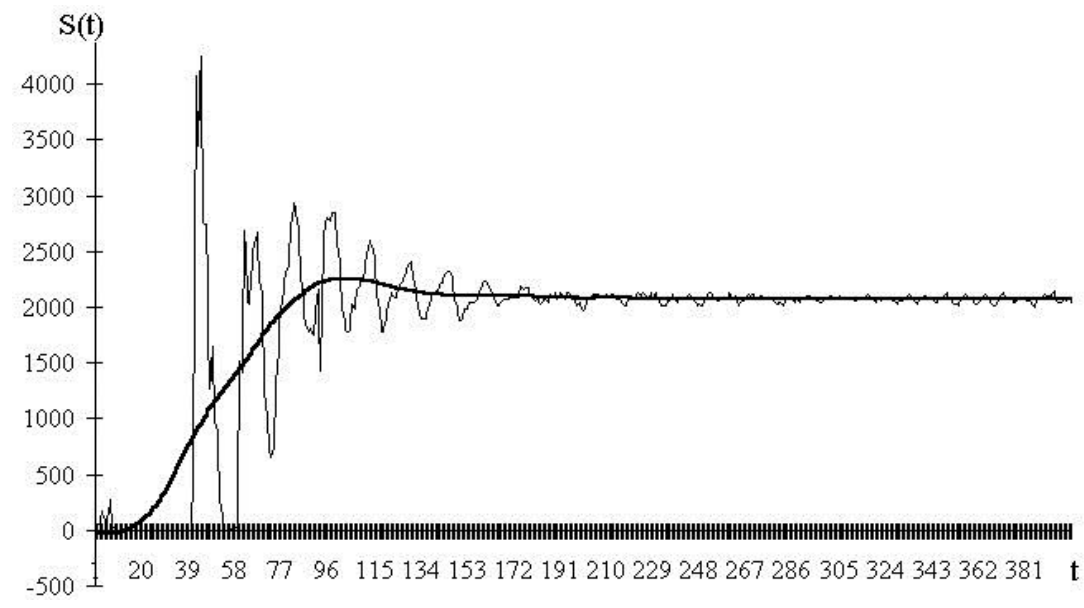

Fig. 10. Transient measurement processing results.

For the case under consideration (Fig. 10) under the assumption that the measured process for 20 counts can be described by a polynomial of the second degree (i.e., the minimum covering length is 20). The processing results of the measurement results, in the availability of a priori information about a small approximation interval, are shown in Fig. 11 , in the case when the researcher is interested in the oscillatory process.

Thus, using the method of useful component propagation allows multifunctional analysis of the measurement results and allows consistently highlight the part of the measured process that has the maximum information content when conducting research.

The synthesis of the MPE method and the moving average method made it possible to implement the method of piecewise multiplication of estimates, which combines both of 
these methods and which allows one to eliminate the end effects of one method and implement a local method for the other $[6,10]$.

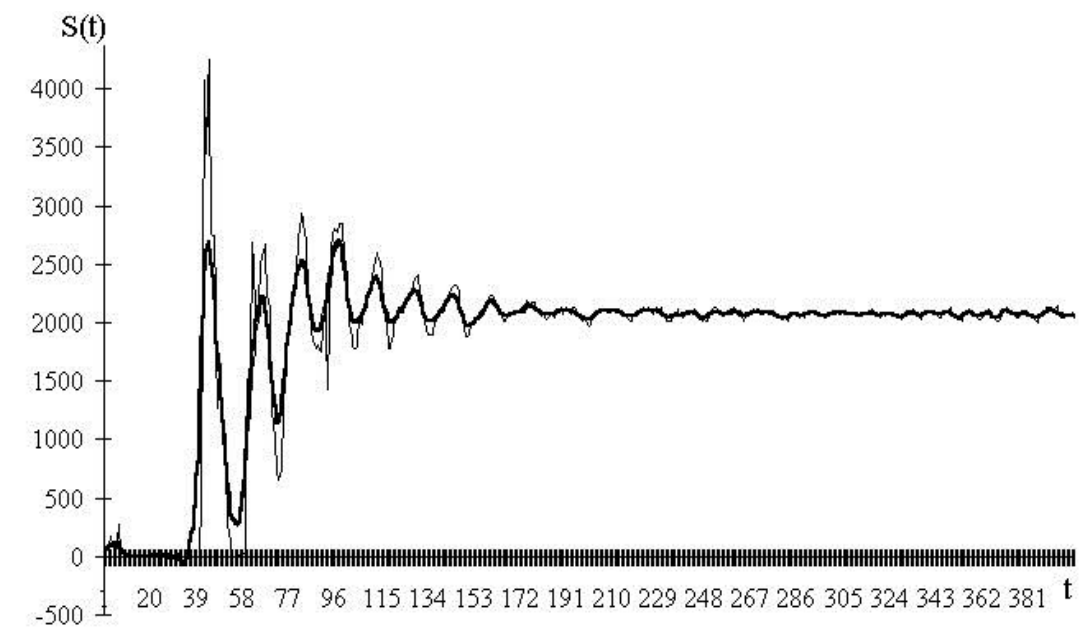

Fig. 11. Transient measurement processing results with a minimum covering length of 20 .

Based on the MPE method, it is possible to implement a method for detecting anomalous measurements, which has very interesting properties. Consider the dependence of the first kind error $\alpha=f(D)$ for various signal models, the results of which are shown in Fig. 12. The number of anomalous measurements is $10 \%$ of the sample size, and their amplitude value $\mathrm{D}$ varies from $2 \sigma_{n}$ to $50 \sigma_{n}$.

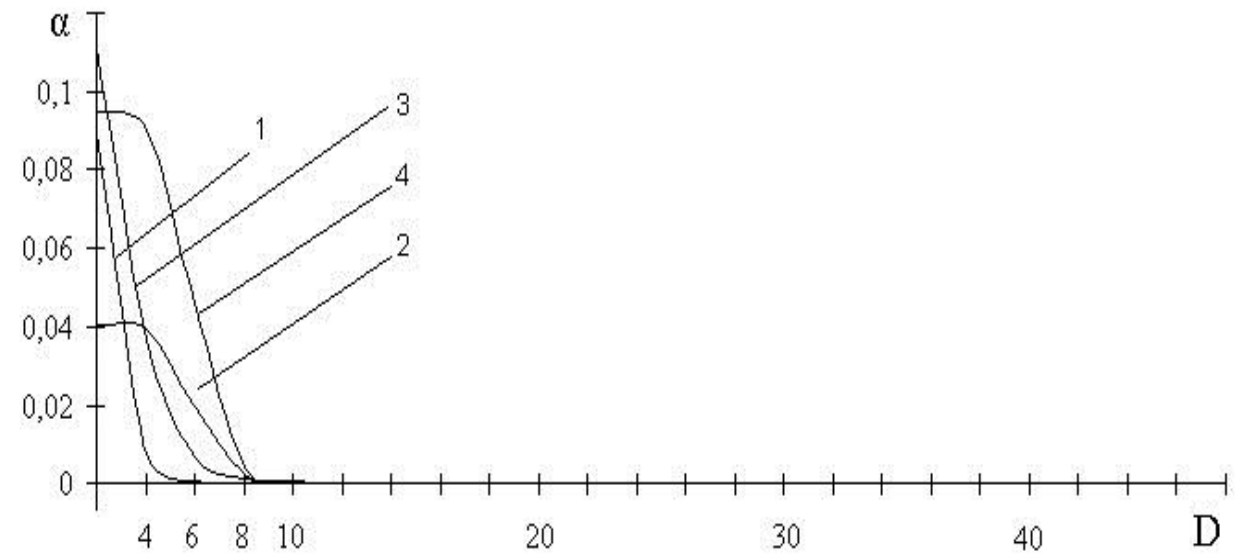

Fig. 12. Dependence $\alpha=f(D)$ for various signal models.

The following components are used as models of the useful component: 1 graph - no model; 2 graph - sinusoidal model; 3 graph - exponential model; 4 graph - a complex model.

An analysis of the obtained graphs shows that, with the amplitude of the anomalous measurements $D \geq 8 \sigma_{n}$ the first kind error, regardless of the model of the useful signal, is almost zero. Special interest is the analysis of the dependence of the probability of correct detection of $\beta=f(D)$ for the same signal models that are shown in Fig. 13. As can be seen from the graphs showed in Fig. 13, for small values of the amplitude of anomalous 
measurements, the probability of correct detection lies in the range from 0.4 to 0.8 . With increasing the anomalous measurements amplitude, the value of $\beta$ increases, and at values of $D \geq 8 \sigma_{n}$ almost reached value 1, regardless of the model of the useful component of the measurement results.

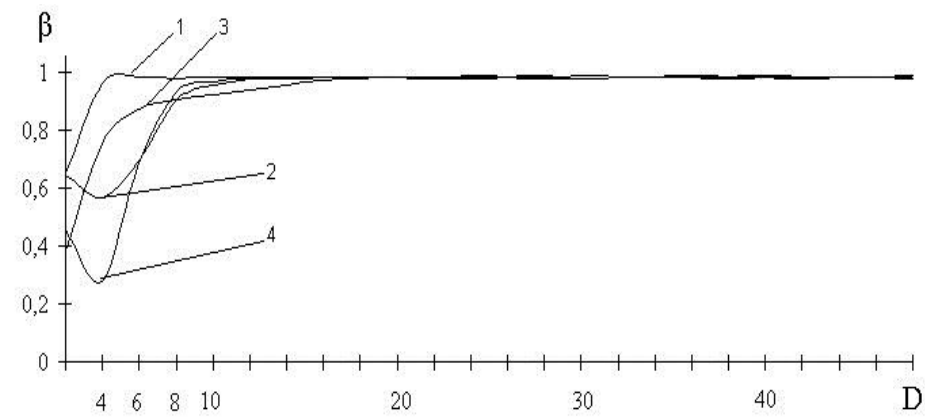

Fig. 13. Dependence $\beta=f(D)$ for various signal models.

Thus, the new method allows us to determine anomalous values in the presence of the signal $\beta \approx 1$ and $a \approx 0$.

The analysis of the given fragment in Fig. 6 shows that alternating values in a row more or less than zero are highlighted and the resulting difference process has a better structure for more accurate estimation of mathematical expectation [11-15]. When comparing the estimates of the mathematical expectation of the initial implementation and the processed one, a fragment of which is presented in Fig. 14, it follows that only in $2 \%$ of the 1000 random simulated process realizations, the ratio of the average without processing to the average after processing $\bar{m}(t) / \bar{m}_{1}(t)$ is less than unity (a straight line in the figure), and in a number cases, this ratio reaches hundreds and thousands of times. A comparative analysis of 1000 implementations with a length of 100 points shows that the average value of the ratio $\bar{m}(t) / \bar{m}_{1}(t)$ is more than 33 .

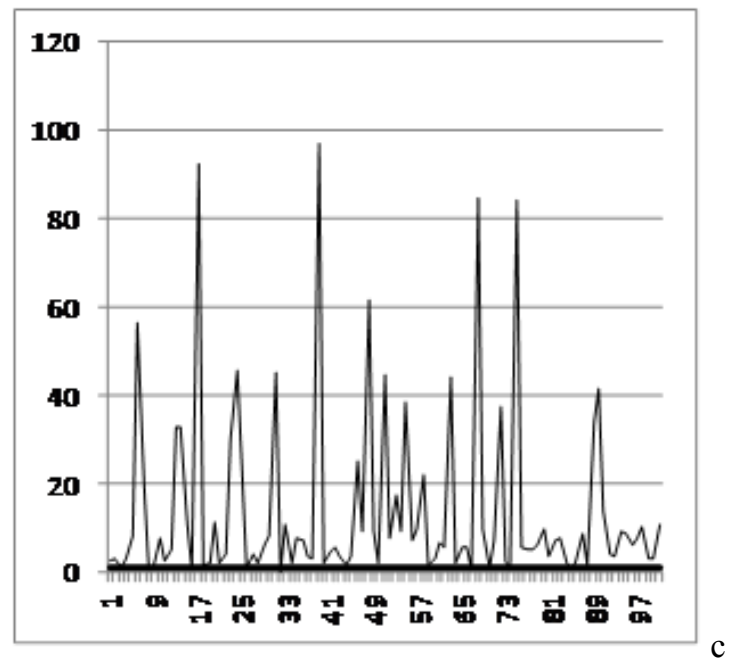

Fig.14. A fragment of comparing the estimates of the mathematical expectation of the original implementation and the processed. 


\section{Conclusions}

The following conclusions can be drawn from the research:

1. Using the method of multiplying estimates of the useful component allows the processing of measurement results with a limited amount of a priori information about the function of the useful component, while the approximation error asymptotically tends to the optimal.

2. The use of the estimation bias compensation technique with the one-sided noise distribution law makes it possible to almost completely compensate for the estimation bias of the useful signal function, while the standard error decreases by more than 4 times.

3. Varying the parameters of the MPE allows you to significantly expand its capabilities when processing measurement results (for example, the analysis of the transition process).

4. The method for detecting anomalous measurements on the basis of the MPE allows one to determine anomalous values in the presence of a signal with $\beta \approx 1$ and $\alpha \approx 0$.

5. The synthesis of the moving average method and MPE allows you to increase processing efficiency and completely eliminate the end effects.

6. The use of MPE for "cleaning" the simulated random process can significantly reduce the error in estimating the mathematical expectation of the sample length (33 times on average).

\section{References}

1. T. Anderson, Statistical analysis of time series. (Mir, Moscow, 1976)

2. .V.I. Peregudov, The Least Squares Method and its Application in Research (Statistics, Moscow, 1965)

3. S.M. Perevertkin, A.V. Kantor, N.F. Borodin, T.S. Shcherbakova, Onboard telemetry equipment of spacecraft (Mashinostroenie, Moscow, 1977)

4. O.N. Novoselov, A.F. Fomin, A.V. Plyushchev, Measurements, Control, Automation, 3-10 (1981)

5. V.P. Vapnik, Algorithms and programs for dependency recovery, (Science, Moscow, 1984)

6. V.I. Marchuk, I.S. Shraifel, Methods of extraction of useful component in a priori uncertainty and a limited volume of measurement results. (SSSU, Shakhty, 2008)

7. V.I. Marchuk, Measuring equipment, 12, 3-5 (2003)

8. V.I. Marchuk, Measurement Techniques. T. 46. № 12, 1121-1126 (2003)

9. V. Marchuk, S. Makov, A. Minaev, V. Voronin, D. Chernyshov, Proceedings of 2016 IEEE East-West Design and Test Symposium, EWDTS 2016, 7807624 (2016).

10. V.I. Marchuk, K.E. Rumyantsev, A.I. Sherstobitov, Radio engineering, 9, 3-7 (2006)

11. V.I. Marchuk, V.V. Voronin, A.I. Sherstobitov, Radio engineering, 9, 75- 82 (2011)

12. V.I. Marchuk, V.V. Voronin, A.I. Sherstobitov, E.A. Semenishchev, Methods of digital signal processing for solving applied problems, (Radio engineering, Moscow, 2012)

13. V.I.Marchuk, Serbian journal of electrical engineering, Vol. 15, № 3, 365-370 (2018)

14. V.I. Marchuk, D. Y. Chernyshov, MATEC Web Conf., 226, 05005 (2018)

15. V.I. Marchuk, A.V. Fisunov, V.B. Gnezdilova, MATEC Web Conf., 226, 05003 (2018) 